\title{
Retrospective Investigation of Cutaneous Squamous Cell Carcinoma on the Lip Treated with Peplomycin Administered Through a Superficial Temporal Artery
}

\author{
TAKU FUJIMURA ${ }^{1 *}$, KAZUHIRO TAKAHASHI $^{2 *}$, YUMI KAMBAYASHI $^{1}$, \\ SADANORI FURUDATE ${ }^{1}$, TAKANORI HIDAKA ${ }^{1}$, AYA KAKIZAKI ${ }^{1}$, AKIKO WATABE $^{1}$, \\ TAKAHIRO HAGA ${ }^{1}$, AKIRA HASHIMOTO ${ }^{1}$ and SETSUYA AIBA ${ }^{1}$ \\ ${ }^{1}$ Department of Dermatology, Tohoku University Graduate School of Medicine, Sendai, Japan; \\ ${ }^{2}$ Department of Dermatology, Iwate Medical University, Morioka, Japan
}

\begin{abstract}
Background: Continuous intra-arterial (IA) administration of peplomycin (PEP) through a tumor-feeding artery is one of the most effective treatments for cutaneous squamous cell carcinoma (cSCC) in cosmetic areas. Patients and Methods: In order to determine the effective and safe dose of PEP and the curative rate of IA-PEP, we retrospectively investigated a case series of 24 patients with cSCC on the lips who were treated with IA-PEP. Results: IA-PEP reduced the tumor mass in all 24 cases (100\%). A complete response occurred in 17 patients $(70.8 \%)$, and a partial response occurred in seven (29.2\%). Moreover, 17 patients (70.8\%) were cured, three patients developed cervical lymph node metastasis (12.5\%), and four developed local recurrence (16.7\%). Three out of the 24 patients developed interstitial pneumonia (12.5\%). Conclusion: Low-dose IA-PEP administered through a superficial temporal artery was a highly effective treatment that achieved a curative response for $70.8 \%$ of patients with cSCC on the lips.
\end{abstract}

Cutaneous squamous cell carcinoma ( $\mathrm{cSCC}$ ) is the second most common type of non-melanoma skin cancer (1). Treatment for cSCC is surgical excision or local radiation for conventional cases (2). With advanced tumors and tumors in cosmetic areas, the management of $\mathrm{cSCC}$ is determined on a

\footnotetext{
*These Authors equally contributed to this work.

Correspondence to: Taku Fujimura, Department of Dermatology, Tohoku University Graduate School of Medicine, 1-1 Seiryo-machi, Aoba-ku, Sendai, Miyagi 980-8574, Japan. Tel: +81 227177271, Fax: +81227177361, e-mail: tfujimura1@mac.com

Key Words: Cutaneous SCC on the lip, peplomycin, continuous intra-arterial administration, curative response, interstitial pneumonia.
}

case-by-case basis $(1,3)$. Among the therapeutic options, chemotherapy is used for the treatment of inoperable cSCC (2, 4). Alter et al. reviewed the therapeutic effects of epidermal growth factor receptor inhibitors, such as cetuximab, for the treatment of advanced cSCC, but the 5-year tumor-free rate (cured patients) was not reported (2). More recently, Magrini et al. reported that cetuximab is more effective than cisplatin for the treatment of advanced cSCC in combination with radiotherapy (5). They concluded that the 3-year overall survival rate is significantly higher in patients with cSCC treated with cetuximab and radiotherapy compared to those treated with cisplatin and radiotherapy. Since drug tolerance is sometimes induced in molecular-targeted therapy (6), the induction of an antitumor immune response is important for the long-term response in patients with cancer (7).

With regard to conventional chemotherapy for $\mathrm{cSCC}$ in cosmetic areas, Edwards et al. reported the therapeutic effect of intralesional injection of interferon alpha-2b, which achieved a high percentage of excellent cosmetic results (93.9\%) (4). DeConti also reviewed chemotherapy for cSCC and reported the efficacy of several combination preoperative chemotherapies (1). The overall response rate of patient to preoperative chemotherapy using cisplatin and bleomycin was $80 \%$, and the objective response rate to combination therapy using cisplatin, 5-fluorouracil and bleomycin was $84.6 \%$ (1). Although various combination therapies for $\mathrm{cSCC}$ have been reported, case series of monotherapies for $\mathrm{cSCC}$ are limited, and the curative rates of these treatments are unknown $(1,2)$. We previously reported that even a single administration of peplomycin (PEP) was useful for the treatment of SCC in a small case series and that increased numbers of cytotoxic T-cells are a possible mechanism for the long-term antitumor effects of this monotherapy (8). Our previous study suggested that antitumor immune responses are induced by the intra-arterial administration of PEP, leading to long-term antitumor responses. In the present 
report, we further retrospectively investigated the efficacy of PEP continuously administered through a superficial temporal artery for the treatment of $\mathrm{cSCC}$ on the lip and evaluated the curative rate of this monotherapy.

\section{Patients and Methods}

Patients. We reviewed a retrospectively collected database of the Department of Dermatology, Tohoku University Graduate School of Medicine, to identify 24 patients with cSCC on the lips that had been treated with intra-arterial administration of PEP through a superficial temporal artery by using an intravascular indwelling catheter from April 1997 through March 2011 (Table I). All patients gave their written informed consent. The study was approved by the Ethics Committee of Tohoku University Graduate School of Medicine, Sendai, Japan (2015-1-491).

Indigo carmine was used to place an indwelling catheter into the facial artery, which is the branch of the superficial temporal artery that fed the tumor (8), and $5 \mathrm{mg}$ per day of PEP was continuously administered through the catheter (Figure 1). Patients were staged according to the criteria of the Union for International Cancer Control classification system (9).

Treatment schedule. Before the administration of PEP, we used computed tomography to screen for possible internal malignancies and interstitial pneumonia in all 24 patients but found none. We then performed continuous intra-arterial administration of PEP through a superficial temporal artery, continuously administering $5 \mathrm{mg}$ per day of PEP for 10 to 12 days through the facial artery. If the patient was suspected of developing severe side-effects, such as interstitial pneumonia or embolization of the catheter, we immediately stopped the administration of peplomycin. Subsequently, we observed the tumor mass for 6 weeks. If tumors were macroscopically and histologically absent, the patients were considered to have a complete response (CR). If tumors remained, patients were considered to have a partial response (PR), and the remaining tumors were excised. A representative case of a patient with cSCC treated with intra-arterial administration of PEP through a superficial temporal artery is shown in Figure 2A-E, and a representative case of surgical treatment of cSCC on the lip is shown in Figure 2F-H.

Response assessment. All patients were followed for at least 5 years. We screened for possible local recurrence with a regular physical examination every 3 months for 5 years (Table II). We screened for possible lymph node metastasis and remote metastasis with a computed tomographic scan once a year for 5 years. Subsequently, 5 years after the administration of peplomycin, if no tumors were present, we regarded these patients as cured.

Statistical methods. Tumor response and side-effect rate, and their 95\% confidence interval (CI) were estimated.

\section{Results}

Demographic data. Patient demographic data are shown in Table I. The patients consisted of 18 men and 6 women with an average age of 72.3 years In all cases, tumors were located on the lower lips. Fifteen cases were stage I, and nine were stage II. Histologically, 11 cases were well-
Table I. Patient demographic data, tumor stage, and histological subtype.

\begin{tabular}{lcccccc}
\hline Case & Age, years & Gender & Location & TMN & Stage & Histology \\
\hline 1 & 88 & M & Lower lip & T1N0M0 & I & WD \\
2 & 81 & M & Lower lip & T2N0M0 & II & MD \\
3 & 70 & F & Lower lip & T1N0M0 & I & WD \\
4 & 70 & M & Lower lip & T1N0M0 & I & MD \\
5 & 59 & M & Lower lip & T2N0M0 & II & MD \\
6 & 66 & M & Lower lip & T2N0M0 & II & PD \\
7 & 61 & F & Lower lip & T1N0M0 & I & WD \\
8 & 62 & M & Lower lip & T1N0M0 & I & MD \\
9 & 64 & M & Lower lip & T1N0M0 & I & MD \\
10 & 71 & M & Lower lip & T2N0M0 & II & MD \\
11 & 75 & M & Lower lip & T2N0M0 & II & PD \\
12 & 79 & M & Lower lip & T2N0M0 & II & MD \\
13 & 88 & M & Lower lip & T3N0M0 & II & WD \\
14 & 59 & M & Lower lip & T3N0M0 & II & MD \\
15 & 69 & F & Lower lip & T1N0M0 & I & MD \\
16 & 81 & F & Lower lip & T1N0M0 & I & WD \\
17 & 72 & M & Lower lip & T1N0M0 & I & WD \\
18 & 67 & M & Lower lip & T1N0M0 & I & WD \\
19 & 77 & F & Lower lip & T1N0M0 & I & WD \\
20 & 73 & M & Lower lip & T1N0M0 & I & MD \\
21 & 75 & M & Lower lip & T1N0M0 & I & WD \\
22 & 62 & M & Lower lip & T1N0M0 & I & MD \\
23 & 87 & M & Lower lip & T3N0M0 & II & WD \\
24 & 79 & F & Lower lip & T1N0M0 & I & WD \\
\hline
\end{tabular}

M: Male; F: female; WD: well-differentiated; MD: moderately differentiated; PD: poorly differentiated.

differentiated SCC, 11 were moderately differentiated SCC, and two were poorly differentiated SCC.

Efficacy of administration of PEP. Twenty-one out of the 24 patients received a complete treatment course of PEP $(87.5 \%$; $95 \% \mathrm{CI}=0-175.0 \%)$. In three patients $(12.5 \%$; $95 \%$ $\mathrm{CI}=0-25.0 \%)$, the administration of PEP was stopped due to adverse events. In all 24 cases (100\%), the tumor size was reduced by intra-arterial administration of PEP. Case 1 is shown as a representative case in Figure 2. Six weeks after the administration of PEP, we evaluated the tumor histologically and macroscopically. In 17 cases, the SCC tumor mass was completely absent $(70.8 \%$; $95 \% \mathrm{CI}=0$ $141.6 \%)$, and in seven cases $(29.2 \%$; $95 \% \mathrm{CI}=0-58.4 \%)$, some tumor mass persisted and was excised. Among our 24 patients, 17 patients $(70.8 \%$; 95\% CI $=0-141.6 \%)$ were cured (5 years tumor-free), three patients developed cervical lymph node metastasis $(12.5 \%$; $95 \% \mathrm{CI}=0-25.0 \%)$, and four developed local recurrence $(16.7 \%$; $95 \% \mathrm{CI}=0-33.4 \%)$.

Side-effects. Among the 24 patients, three developed interstitial pneumonia $(12.5 \%$; $95 \% \mathrm{CI}=0-25.0 \%)$. There were no other grade 3-5 side-effects. 


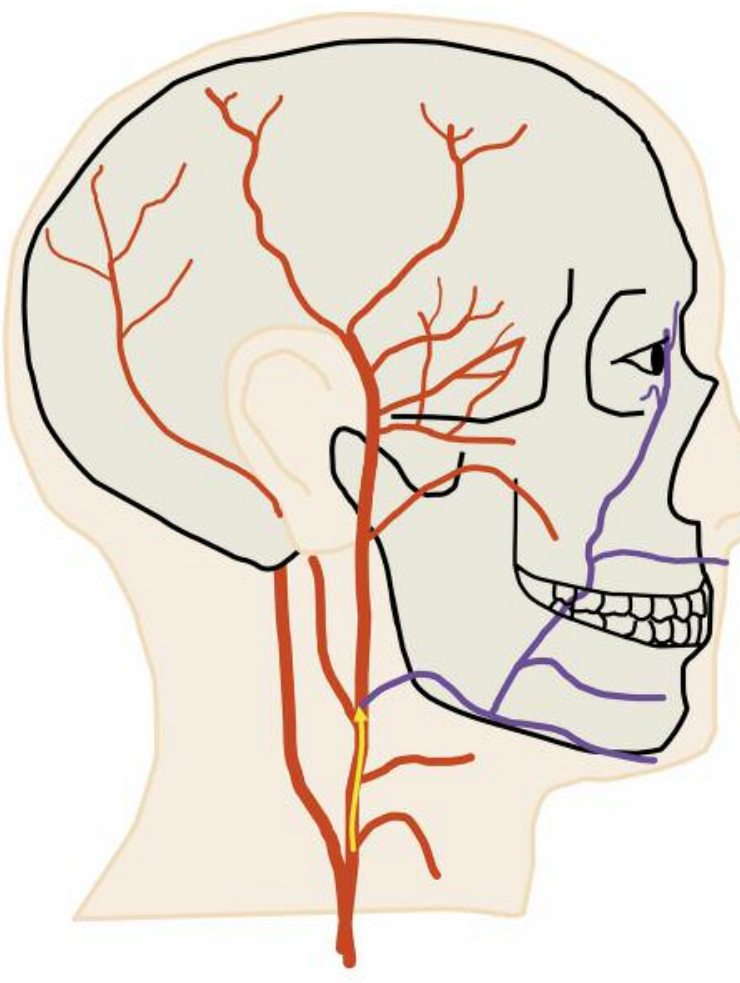

Figure 1. Schema of intra-arterial administration of peplomycin through a superficial temporal artery. We placed the indwelling catheter into the facial artery (yellow arrow), which is a branch of the superficial temporal artery. We then continuously administered $5 \mathrm{mg}$ peplomycin per day (purple line).

\section{Discussion}

PEP is a bleomycin derivative antibiotic that is used as preoperative chemotherapy for SCC in Japan (10-12). The total dose for conventionally administered PEP ranges from 20 to $110 \mathrm{mg}$ by bolus injection of $5-10 \mathrm{mg} /$ day $(10,11)$. The dose of PEP is limited due to dose-dependent fatal sideeffects, such as interstitial pneumonia (10-12). Therefore, in order to achieve a high concentration of PEP only in the tumor site, we selected continuous intra-arterial administration of PEP through a superficial temporal artery for the treatment of $\operatorname{cSCC}(8,13)$. In addition, as we previously reported, continuous intra-arterial administration of PEP increased the number of cytotoxic T-cells in the tumor site of cSCC, suggesting that this monotherapy might be suitable for the induction of a long-term anti-tumor response through the host immune system. Accordingly, in the present report, we investigated the efficacy of PEP continuously administered through a superficial temporal artery for the treatment of cSCC on the lip, and retrospectively evaluated the curative rate of this monotherapy.
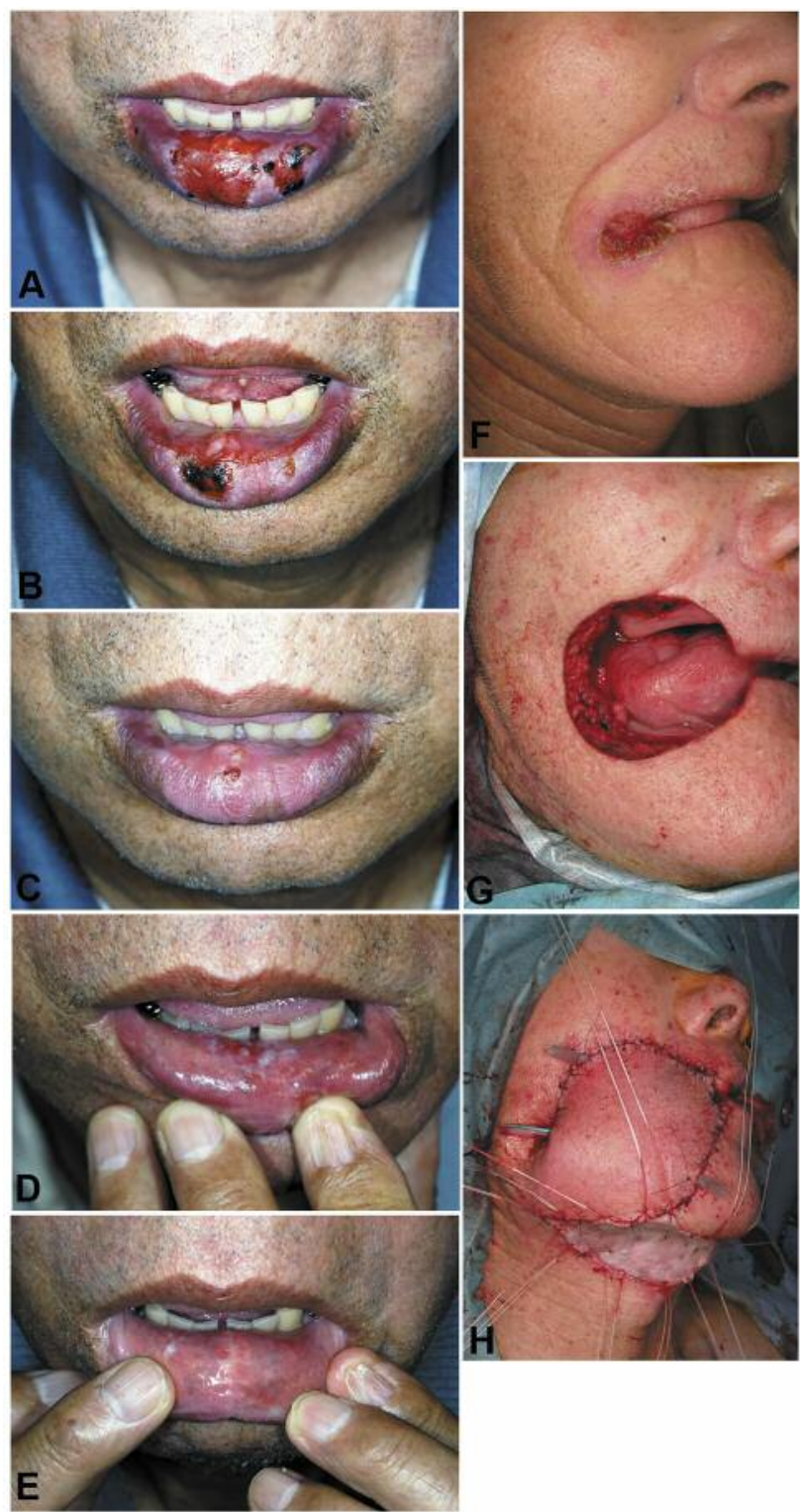

Figure 2. Cutaneous squamous cell carcinoma (SCC) on the lip treated with continuous intra-arterial administration of peplomycin $(A-E)$ or conventional surgery $(F-H)$. Appearance of case 1 at day $0(A), 7(B)$, $21(C), 56(D)$ and 84 (E) after completion of continuous, intra-arterial administration of $5 \mathrm{mg}$ per day for cutaneous SCC of the lip. Case of cutaneous SCC treated with conventional surgical methods: before treatment $(F)$, radical excision $(G)$, and reconstruction by skin-flap $(H)$.

In our present study, intra-arterial administration of PEP dramatically reduced the tumor mass in all 24 cases of cSCC on the lip. Notably, with conventional surgical methods, radical excision and skin-flap is mandatory in treating $\mathrm{cSCC}$ on the lip, as shown in Figure 2F-H. On the other hand, only inconspicuous scars remained after treatment with 
Table II. Patient demographic data, dose of peplomycin, response evaluation, prognosis, and side-effects.

\begin{tabular}{|c|c|c|c|c|c|c|}
\hline Case & Age, years & Dose & Response & Prognosis & Side-effect & Resection \\
\hline 1 & 88 & $50 \mathrm{mg}$ & PR & 5 Years tumor-free & - & $2 \mathrm{M}$ \\
\hline 2 & 81 & $50 \mathrm{mg}$ & $\mathrm{CR}$ & 5 Years tumor-free & - & - \\
\hline 3 & 70 & $60 \mathrm{mg}$ & $\mathrm{CR}$ & 5 Years tumor-free & - & - \\
\hline 4 & 70 & $37.5 \mathrm{mg}$ & $\mathrm{CR}$ & 5 Years tumor-free & - & - \\
\hline 5 & 59 & $60 \mathrm{mg}$ & $\mathrm{CR}$ & Lymph node metastasis & - & - \\
\hline 6 & 66 & $60 \mathrm{mg}$ & $\mathrm{CR}$ & Local recurrence & - & - \\
\hline 7 & 61 & $60 \mathrm{mg}$ & $\mathrm{CR}$ & 5 Years tumor-free & - & - \\
\hline 8 & 62 & $55 \mathrm{mg}$ & $\mathrm{CR}$ & 5 Years tumor-free & - & - \\
\hline 9 & 64 & $50 \mathrm{mg}$ & $\mathrm{CR}$ & 5 Years tumor-free & - & - \\
\hline 10 & 71 & $50 \mathrm{mg}$ & $\mathrm{CR}$ & Local recurrence & - & - \\
\hline 11 & 75 & $30 \mathrm{mg}$ & $\mathrm{CR}$ & 5 Years tumor-free & - & - \\
\hline 12 & 79 & $60 \mathrm{mg}$ & $\mathrm{CR}$ & 5 Years tumor-free & - & - \\
\hline 13 & 88 & $50 \mathrm{mg}$ & $\mathrm{CR}$ & 5 Years tumor-free & - & - \\
\hline 14 & 59 & $50 \mathrm{mg}$ & $\mathrm{CR}$ & Local recurrence & - & - \\
\hline 15 & 69 & $50 \mathrm{mg}$ & $\mathrm{CR}$ & 5 Years tumor-free & - & - \\
\hline 16 & 81 & $60 \mathrm{mg}$ & PR & 5 Years tumor-free & - & $2 \mathrm{M}$ \\
\hline 17 & 72 & $60 \mathrm{mg}$ & $\mathrm{CR}$ & Local recurrence & - & - \\
\hline 18 & 67 & $60 \mathrm{mg}$ & PR & 5 Years tumor-free & - & $2 \mathrm{M}$ \\
\hline 19 & 77 & $60 \mathrm{mg}$ & PR & 5 Years tumor-free & Interstitial pneumonia & $2 \mathrm{M}$ \\
\hline 20 & 73 & $60 \mathrm{mg}$ & $\mathrm{CR}$ & 5 Years tumor-free & - & - \\
\hline 21 & 75 & $60 \mathrm{mg}$ & $\mathrm{CR}$ & 5 Years tumor-free & Interstitial pneumonia & - \\
\hline 22 & 62 & $60 \mathrm{mg}$ & PR & Lymph node metastasis & - & $1 \mathrm{M}$ \\
\hline 23 & 87 & $60 \mathrm{mg}$ & PR & Lymph node metastasis & - & $2 \mathrm{M}$ \\
\hline 24 & 79 & $20.5 \mathrm{mg}$ & PR & 5 Years tumor-free & Interstitial pneumonia & $1 \mathrm{M}$ \\
\hline
\end{tabular}

CR: Complete response; PR: partial response; M: month.

continuous intra-arterial administration of PEP (Figure 2AE). At least in terms of cosmetic results, continuous intraarterial administration of PEP is useful for the treatment of cSCC on the lip. Concerning the patient tolerance of this method, 21 out of the 24 patients received a complete course of $\operatorname{PEP}(87.5 \%$; 95\% CI=0-175.0\%). All three patients who did not receive a complete course were cured (tumor-free for 5 years) by subsequent surgical resection. Of the 21 patients that received more than $50 \mathrm{mg}$ of PEP, 14 patients were cured $(66.7 \%$; 95\% CI=0-133.4\%). Concerning the initial tumor response rate, among the 17 cured patients, 12 patients had achieved CR (70.6\%; 95\% CI=0-141.2\%), and five had achieved PR (29.4\%; 95\% CI=0-58.8\%), whereas among the seven patients who experienced relapse, five had achieved CR $(71.4 \% ; 95 \% \mathrm{CI}=0-142.8 \%)$ and 2 patients had achieved PR (28.6\%; 95\% CI $=0-57.2 \%)$. The administration of PEP was stopped in three cases $(12.5 \%$; $95 \% \mathrm{CI}=0-25.0 \%)$ due to adverse events. These data suggest that intra-arterial administration of PEP through a superficial temporal artery was effective for $\mathrm{cSCC}$ on the lips even at a low dose.

Concerning the three patients who developed interstitial pneumonia, one had achieved CR (33.3\%), and all three were cured $(100 \%)$. Among the 21 patients without interstitial pneumonia, 16 patients $(76.2 \%$; $95 \% \mathrm{CI}=0-152.4 \%)$ achieved CR, five (23.8\%; 95\% CI=0-47.6\%) achieved PR, and 14 patients $(66.7 \%$; 95\% CI $=0-133.4 \%)$ were cured. The occurrence rate of interstitial pneumonia was low $(12.5 \%$; $95 \%$ $\mathrm{CI}=0-25.0 \%$ ), and the patients that developed interstitial pneumonia had a good response to intra-arterial PEP therapy. Notably, several experimental reports have suggested that bleomycin-induced lung injury might be enhanced by the reduction of regulatory T-cells (Tregs) in the circulation (1417). For example, Nemoto et al. reported the protective effect of Tregs and acceleration effects of type 1 helper cells, and type 17 helper cells, on bleomycin-induced lung injury in a mouse model (16). On the other hand, several clinical reports have suggested the contribution of tumor-infiltrating forkhead box P3-positive Tregs on the establishment and progression of tumor (18-21). Notably, as we previously reported, intra-arterial administration of PEP reduced the ratio of Tregs among tumorinfiltrating leukocytes (8), which further suggests that PEP might systemically reduce Tregs and promote interstitial pneumonia. Our present cases and these previous reports suggest the possible correlation between the induction of an antitumor immune response and the occurrence of interstitial pneumonia by the administration of PEP. Since we did not assess the ratio of Tregs in peripheral blood in the present study, further studies are needed to test our hypothesis.

In the present report, we describe a case series of 24 patients with cSCC who were successfully treated with 
continuous intra-arterial administration of PEP through a superficial temporal artery. Our present data suggest that such therapy is effective for cSCC on the lips, even at a low dose, and that interstitial pneumonia occurred without dose dependency. Our data suggest that although intra-arterial administration of PEP is one of the most effective treatments for $\mathrm{cSCC}$ in cosmetic areas, dermato-oncologists should take into account the occurrence of interstitial pneumonia even with use of a low dose of PEP.

\section{Conflicts of Interest}

The Authors have no conflicts of interest to declare.

\section{Funding}

This study was supported in part by grants-in-aid for scientific research from the Japan Society for the Promotion of Science (16K10143).

\section{Ethics Approval}

This study was approved by the Ethics Committee of Tohoku University Graduate School of Medicine, Sendai, Japan (2015-1-491).

\section{References}

1 DeConti RC: Chemotherapy of squamous cell carcinoma of the skin. Semin Oncol 39: 145-149, 2012.

2 Alter M, Satzger I, Mattern A, Kapp A and Gutzmer R: Treatment of advanced cutaneous squamous cell carcinomas with epidermal growth factor receptor inhibitors. Dermatology 227: 289-294, 2013.

3 Lansbury L, Bath-Hextall F and Perkins W, Stanton W and Leonardi-Bee $\mathrm{J}$ : Interventions for non-metastatic squamous cell carcinoma of the skin: systematic review and pooled analysis of observational studies. BMJ 347: f6153, 2013.

4 Edwards L, Berman B, Rapini RP, Whiting DA, Tyring S, Greenway HT Jr, Eyre SP, Tanner DJ, Taylor EL, Peets E and Smiles KA: Treatment of cutaneous squamous cell carcinomas by intralesional interferon $\alpha-2 b$ therapy. Arch Dermatol 128: 1486-1489, 1992.

5 Magrini SM, Buglione M, Corvò R, Pirtoli L, Paiar F, Ponticelli P, Petrucci A, Bacigalupo A, Crociani M, Lastrucci L, Vecchio S, Bonomo P, Pasinetti N, Triggiani L, Cavagnini R, Costa L, Tonoli S, Maddalo $\mathrm{M}$ and Grisanti S: Cetuximab and radiotherapy versus cisplatin and radiotherapy for locally advanced head and neck cancer: A Randomized Phase II Trial. J Clin Oncol 34: 427-435, 2016.

6 Soura E, Chasapi V and Stratigos AJ: Pharmacologic treatment options for advanced epithelial skin cancer. Expert Opin Pharmacother 16: 1479-1493, 2015.

7 Winkler JK, Schneiderbauer R, Bender C, Enk A and Hassel JC: PD-1 blockade: a therapeutic option for treatment of metastatic Merkel cell carcinoma. Br J Dermatol 176: 216-219, 2016.

8 Fujimura T, Kambayashi Y, Furudate S, Kakizaki A, Haga T, Hashimoto A and Aiba S: Immunomodulatory effects of peplomycin on immunosuppressive and cytotoxic cells in the lesional skin of cutaneous squamous cell carcinoma. Dermatology 230: 250-255, 2015.

9 Gospodarowicz M, Wittekind C and Sobin L: TNM Classification of Malignant Tumor, Seventh Edition. Union for International Cancer Control pp. 32-35, 2010.

10 Kirita T, Shimooka H, Yamanaka Y, Tatebayashi S, Yamamoto $\mathrm{K}$, Nishimine $\mathrm{M}$ and Sugimura $\mathrm{M}$ : Prognostic value of response to preoperative chemoradiotherapy and residual tumor grades in tongue carcinoma. Oral Surg Oral Med Oral Pathol Oral Radiol Endod 91: 293-300. 2001.

11 Isobe K, Uno T, Hanazawa T, Kawakami H, Yamamoto S, Suzuki $\mathrm{H}$, Iida $\mathrm{Y}$, Ueno $\mathrm{N}$, Okamoto $\mathrm{Y}$ and Ito $\mathrm{H}$ : Preoperative chemotherapy and radiation therapy for squamous cell carcinoma of the maxillary sinus. Jpn J Clin Oncol 35: 633-638, 2005.

12 Sugiyama T, Hasuo Y, Nishida $\mathrm{T}$ and Kamura T: Impact on survival following successful neoadjuvant chemotherapy and radical surgery for Stage IIb bulky and Stage IIIb cervical cancer. Gynecol Oncol 81: 330-331, 2001.

13 Haga T, Fujimura T, Takeuchi I, Deguchi $M$ and Aiba S: Successful treatment of two cases of squamous cell carcinoma on the ear with intra-arterial administration of peplomycin through superficial temporal artery. Case Rep Dermatol 6: 207-211, 2014.

14 Boveda-Ruiz D and D'Alessandro-Gabazza CN and Toda M, Takagi T, Naito M, Matsushima Y, Matsumoto T, Kobayashi T, Gil-Bernabe P, Chelakkot-Govindalayathil AL, Miyake Y, Yasukawa A, Morser J, Taguchi O and Gabazza EC: Differential role of regulatory $\mathrm{T}$ cells in early and late stages of pulmonary fibrosis. Immunobiology 218: 245-254, 2013.

15 Tang YJ, Xiao J, Huang XR, Zhang Y, Yang C, Meng XM, Feng YL, Wang XJ, Hui DS, Yu CM and Lan HY: Latent transforming growth factor- $\beta 1$ protects against bleomycin-induced lung injury in mice. Am J Respir Cell Mol Biol 51: 761-771, 2014.

16 Nemoto Y, Kanai T, Takahara M, Oshima S, Okamoto R, Tsuchiya K, Matsumoto S and Watanabe M: Th1/Th17-mediated interstitial pneumonia in chronic colitis mice independent of intestinal microbiota. J Immunol 190: 6616-6625, 2013.

17 Galati D, De Martino M, Trotta A, Rea G, Bruzzese D, Cicchitto G, Stanziola AA, Napolitano M, Sanduzzi A and Bocchino M: Peripheral depletion of NK cells and imbalance of the Treg/Th17 axis in idiopathic pulmonary fibrosis patients. Cytokine 66: 119126, 2014.

18 Kambayashi $\mathrm{Y}$, Fujimura $\mathrm{T}$ and Aiba $\mathrm{S}$ : Comparison of immunosuppressive cells and immunomodulatory cells in kerartoacanthoma and invasive squamous cell carcinoma. Acta Derm Venereol 93: 663-668, 2013.

19 Tallon B and Bhawan J: FOXP3 expression is increased in cutaneous squamous cell carcinoma with perineural invasion. $\mathrm{J}$ Cutan Pathol 37: 1184-1185, 2010.

20 Strauss L, Bergmann C, Gooding W, Johnson JT and Whiteside TL: The frequency and suppressor function of $\mathrm{CD} 4{ }^{+} \mathrm{CD} 25^{\text {high }} \mathrm{FOXP} 3^{+}$ T-cells in the circulation of patients with squamous cell carcinoma of the head and neck. Clin Cancer Res 13: 6301-6311, 2007.

21 Tabachnyk M, Distel LV, Büttner M, Grabenbauer GG, Nkenke E, Fietkau R and Lubgan D: Radiochemotherapy induces a favourable tumour-infiltrating inflammatory cell profile in head and neck cancer. Oral Oncol 48: 594-601, 2012.

Received February 17, 2017 Revised March 8, 2017 Accepted March 13, 2017 